\title{
Five-dimensional regular black holes in a brane world
}

\author{
Juliano C. S. Neves $\odot^{*}$ \\ Instituto de Ciência e Tecnologia, Universidade Federal de Alfenas, \\ Rodovia José Aurélio Vilela, 11999, CEP 37715-400 Poços de Caldas, MG, Brazil
}

(Received 13 July 2021; accepted 16 September 2021; published 4 October 2021)

\begin{abstract}
Following a recent approach, complete and analytic solutions (brane and bulk) of regular black holes are shown in a brane context. The metrics are regular both on the four-dimensional brane and in the fivedimensional bulk. Like many braneworld scenarios, the bulk spacetime is asymptotically anti-de Sitter. On the other hand, a de Sitter core on the brane avoids the singularity inside the event horizon, providing then well-known regular black holes on the brane. From the bulk perspective, the regular black holes are fivedimensional objects, with the event horizon extending to the extra dimension, but the de Sitter core is entirely on the four-dimensional brane.
\end{abstract}

DOI: $10.1103 /$ PhysRevD.104.084019

\section{INTRODUCTION}

The Randall-Sundrum model I [1] was conceived of as an attempt to solve the hierarchy problem in particle physics by using a finite extra dimension and two branes (in which our universe is a brane) embedded into a fivedimensional spacetime. On the other hand, the RandallSundrum II [2] adopts just one brane, with an infinite extra dimension and a warped factor in the bulk or fivedimensional spacetime that, in the second model version, confines gravity close to our universe. Since then such models were studied in other contexts like gravitation and cosmology. ${ }^{1}$ The existence of either a finite or an infinite extra dimension in a context in which our four-dimensional world, a brane, is embedded into a five-dimensional bulk creates new phenomena and has gained attention of many researchers in the last decades.

In gravitation, after the so-called black string from Hawking et al. [5], a large amount of articles exploring the Randall-Sundrum models and the gravitational phenomenon in that context discusses black holes and wormholes. We can divide the area into two approaches: from the brane or from the bulk. In the first one, black hole and wormhole geometries are built from the four-dimensional brane, and equations in the five-dimensional bulk are ignored [6-15]. The second one builds spacetimes from

juliano.neves@ unifal-mg.edu.br

${ }^{1}$ See Refs. $[3,4]$ for a review on brane world scenario.

Published by the American Physical Society under the terms of the Creative Commons Attribution 4.0 International license. Further distribution of this work must maintain attribution to the author(s) and the published article's title, journal citation, and DOI. Funded by SCOAP. the bulk and tries to find out the brane equations [16]. The approach adopted here is the second one.

In a recent work, Nakas and Kanti [17] presented an approach in which the geometry in the bulk is analytically obtained, and then the brane spacetime metric (a known solution in general relativity) and the energy-momentum tensor (an effective tensor, for example) are fully calculated in a Randall-Sundrum II-type model. The main accomplishment of the Nakas-Kanti approach-from bulk to the brane-is to obtain a well-known black hole solution on the brane, like the Schwarzschild geometry Ref. [17]. This will be the approach adopted here. But instead of a black hole with a constant mass like the Nakas and Kanti fivedimensional black holes [17,18], I use a mass function in order to generate a regular black hole $(\mathrm{RBH})$ or a class of RBHs. Contrary to the black string, which is singular in the bulk, or the recent geometries of Nakas and Kanti [17,18], which are regular in the bulk but they are singular on the brane, the geometry (or geometries) presented here is regular in both spacetimes. In particular, the study of the model on the brane will be feasible due to the induced gravitational field equations proposed by Shiromizu, Maeda and Sasaki [19]. The induced field equations provide an effective energy-momentum tensor on the brane. As we will see, the brane spacetime is a vacuum spacetime, and the origin of the RBH on the brane is due to the bulk influence on the brane.

Like the Nakas and Kanti geometry [17], the RBH presented here is a five-dimensional black hole, that is to say, its event horizon extends to the five-dimensional bulk. Moreover, in order to support a RBH, there is a de Sitter core inside the event horizon, just on the brane spacetime, responsible for avoiding the singularity issue. A de Sitter core is, for many RBH solutions, the main feature of such objects [20-25]. 
RBHs have been studied since the pioneer work of Bardeen [20]. This class of black holes, like a good black hole, presents horizons (an inner and an outer horizon) and precludes the central singularity. As I said, a de Sitter core avoids the singularity, then the spacetime of RBHs is entirely regular. The reason for that is some violation of energy conditions inside such objects. With energy conditions banned, the singularity theorems are not valid anymore. ${ }^{2}$ Thus the existence of a singularity inside a $\mathrm{RBH}$ is not a necessary consequence from a mathematical theorem.

The bibliography on RBHs has increased in the last years. Whether in the general relativity context [20-25,27-36] or in other contexts [37-39], RBHs have been studied. Even in the brane world context, there are articles that calculate and explore spacetime metrics of RBHs $[8,15]$. Here, the spacetime geometry is obtained both in the bulk and on the brane. Contrary to previous articles, the complete and analytic solution is then presented, corresponding to known four-dimensional geometries on the brane, like the Bardeen RBH.

This article is structured as follows: in Sec. II one presents the Nakas and Kanti approach and applies it to RBHs, generating then a five-dimensional RBH. In Sec. III the bulk energy-momentum tensor is studied and is shown that energy conditions are violated. In Sec. IV the gravitational field equations are fully calculated, and it is shown that, even with a four-dimensional RBH on the brane, this spacetime is a vacuum spacetime. The final remarks are given in Sec. V.

In this work, geometrized units are adopted. Then $G=c=1$ throughout this article. Capital Latin index runs from 0 to 4 , and Greek index runs from 0 to 3 .

\section{THE BULK PERSPECTIVE}

\section{A. Five-dimensional regular black hole}

The five-dimensional metric in the Randall-Sundrum model II [2] is given by

$$
d s^{2}=e^{-2 k|y|}\left(-d t^{2}+d \vec{x}^{2}\right)+d y^{2},
$$

where $k$ is related to the anti-de Sitter curvature radius $\ell_{\text {AdS }}$ by $k=1 / \ell_{\text {Ads }}$. The five-dimensional bulk $\mathcal{M}$ is asymptotically anti-de Sitter, and the four-dimensional brane $\Sigma$ is located at $y=0$. In the flat coordinates, i.e., when the metric (1) is conformally flat, one has

$$
d s^{2}=\frac{1}{(1+k|z|)^{2}}\left(-d t^{2}+d r^{2}+r^{2} d \Omega_{2}^{2}+d z^{2}\right),
$$

where the new coordinate $z=\operatorname{sgn}(y)\left(e^{k|y|}-1\right) / k$ is adopted (note that the brane is still located at $z=0$ ),

\footnotetext{
${ }^{2}$ See, for example, Ref. [26], chapter 9, for a detailed study on the singularity theorems.
}

and $d \Omega_{2}^{2}=d \theta^{2}+\sin ^{2} \theta d \phi^{2}$ is the line-element of a unit two-sphere.

As I said, the Nakas-Kanti approach [17] to obtain both the bulk metric and the brane metric will be used here. According to those authors, the next step from the general metric (2) regards to impose the spherical symmetry in the bulk. For that purpose, the following change of coordinates is necessary:

$$
r=\rho \sin \chi \quad \text { and } \quad z=\rho \cos \chi,
$$

with $\chi \in[0, \pi]$. With those coordinate transformations, the metric (2) now is written as

$$
d s^{2}=\frac{1}{(1+k \rho|\cos \chi|)^{2}}\left(-d t^{2}+d \rho^{2}+\rho^{2} d \Omega_{3}^{2}\right),
$$

where $d \Omega_{3}^{2}$ plays the role of the line-element from a unit three-sphere, that is to say,

$$
d \Omega_{3}^{2}=d \chi^{2}+\sin ^{2} \chi d \theta^{2}+\sin ^{2} \chi \sin ^{2} \theta d \phi^{2} .
$$

And the inverse transformations of (3) are given by

$$
\rho=\left(r^{2}+z^{2}\right)^{\frac{1}{2}} \quad \text { and } \quad \tan \chi=\frac{r}{z} .
$$

The new radial coordinate $\rho$ is a mix of the bulk extra coordinate and the brane radial coordinate $r$, it ranges from 0 to $\infty$. The coordinate $\chi$ indicates the "left" and "right" side of the brane (see Fig. 1). For $[0, \pi / 2$ [, one has the "right" side (positive values of $z$ ), and for $] \pi / 2, \pi]$, the "left" one (or negative values of $z$ ). Due to the $\mathbb{Z}_{2}$ symmetry in the bulk, points $z$ and $-z$ are equivalent. Therefore, for some calculations, I will rule out the modulus of $y$ in Eq. (4).

Following the famous work on the black string [5], Nakas and Kanti [17] replaced the part of the line

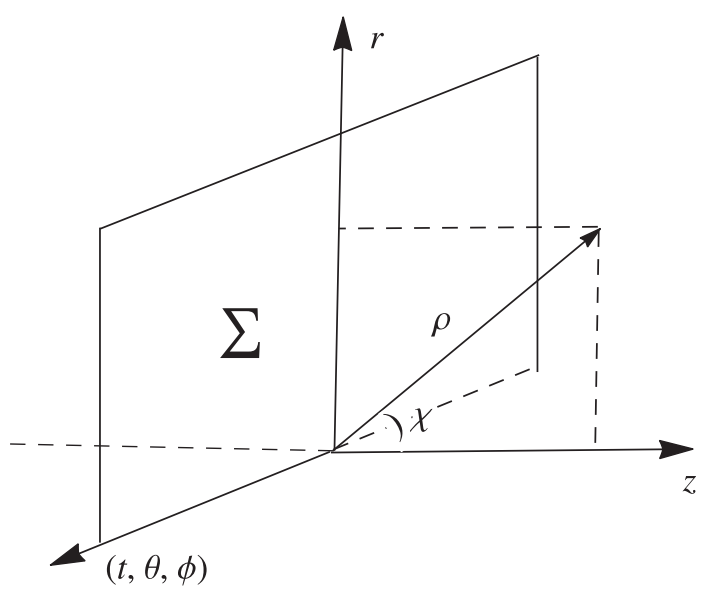

FIG. 1. Brane world context: the four-dimensional brane is indicated as $\Sigma$, described by the $(t, r, \theta, \phi)$ coordinates and is located at $z=0$. It is embedded into the five-dimensional bulk. 
element (4), namely $-d t^{2}+d \rho^{2}$, by the metric elements of the Schwarzschild geometry. Thus, the metric (4) assumed the following form:

$$
d s^{2}=\frac{1}{(1+k \rho \cos \chi)^{2}}\left(-f(\rho) d t^{2}+\frac{d \rho^{2}}{f(\rho)}+\rho^{2} d \Omega_{3}^{2}\right) .
$$

But in order to build a five-dimensional RBH (or an entire class of RBHs), I consider a mass function instead of a constant mass. That is, the metric element $f(\rho)$ is then written as

$$
f(\rho)=1-\frac{2 M(\rho)}{\rho} .
$$

An appropriate mass function that produces RBHs (as we will see) can be written as

$$
M(\rho)=\frac{M_{0}}{\left[1+\left(\frac{r_{0}}{\rho}\right)^{q}\right]^{\frac{3}{q}}} .
$$

Such a mass function generated RBHs in the general relativity context. It is worth mentioning that the mass function (9) provided solutions of Einstein's field equations by using the so-called Synge g-method, in which from a given metric (with, for example, the mass function (9)), one obtains and interprets the resulting energy-momentum tensor (see Ref. [40] for more details on the Synge method). Introduced in Ref. [24], the mass function (9) is able to produce RBHs with and without rotation. In particular, for $q=2$ we have the Bardeen RBH [20], and for $q=3$ the Hayward RBH [29] is obtained. The constant $M_{0}$ is conceived of as the black hole mass, and $q$ is a positive integer in the mass function. On the other hand, $r_{0}$ is adequate to-at least-two interpretations. For $q=2$, the constant $r_{0}$ is interpreted as a charge in a nonlinear electrodynamics according to Ayón-Beato and Garcia's work [41]. But in our work [42], $r_{0}$ is conceived of as length, related to the Planck length. Even an upper bound on $r_{0}$ was assumed but in the general relativity context. ${ }^{3}$ However, as we will see, a mass function like (9) is also able to provide RBHs in a brane context assuming that $r_{0}$ is a short length.

The capability of $M(\rho)$ removing the central singularity is made clear from the Ricci scalar $R$ and from $R_{M N} R^{M N}$ and $R_{M N L K} R^{M N L K}$ (where the last one is also called Kretschmann scalar):

$$
\lim _{\rho \rightarrow 0} R=-20 k^{2}+\frac{40 M_{0}}{r_{0}^{3}},
$$

\footnotetext{
${ }^{3}$ The upper bound obtained in Ref. [42] was $r_{0}<10^{-25} \mathrm{~m}$.
}

$$
\begin{array}{r}
\lim _{\rho \rightarrow 0} R_{M N} R^{M N}=80 k^{4}-\frac{320 M_{0}}{r_{0}^{3}}\left(k^{2}-\frac{M_{0}}{r_{0}^{3}}\right), \\
\lim _{\rho \rightarrow 0} R_{M N L K} R^{M N L K}=40 k^{4}-\frac{160 M_{0}}{r_{0}^{3}}\left(k^{2}-\frac{M_{0}}{r_{0}^{3}}\right) .
\end{array}
$$

As we can see, all results are $q$-independent. Also the metric elements are regular from that mass function, i.e., $g_{t t}=-g_{r r}=-1$ and $g_{\theta \theta}=g_{\phi \phi}=g_{\chi \chi}=0$ for $\rho \rightarrow 0$.

On the other hand, the anti-de Sitter behavior of (7) is indicated as $\rho \rightarrow \infty$. That is,

$$
\begin{gathered}
\lim _{\rho \rightarrow \infty} R=-20 k^{2}, \\
\lim _{\rho \rightarrow \infty} R_{M N} R^{M N}=80 k^{4}, \\
\lim _{\rho \rightarrow \infty} R_{M N L K} R^{M N L K}=40 k^{4} .
\end{gathered}
$$

Therefore, even with a mass function in Eq. (7), the bulk is still asymptotically anti-de Sitter.

In order to better illustrate the event horizon and then present the five-dimensional bulk $\mathrm{RBH}$, one writes the metric (7) in the original $(t, r, \theta, \phi, y)$ coordinates. Thus

$$
\begin{aligned}
d s^{2}= & e^{-2 k|y|}\left[-f(r, y) d t^{2}+\left(\frac{r^{2}}{f(r, y)}+z(y)^{2}\right)\right. \\
& \times \frac{d r^{2}}{r^{2}+z(y)^{2}}+\left(\frac{1}{f(r, y)}-1\right) \frac{2 r z(y) e^{k|y|}}{r^{2}+z(y)^{2}} d r d y \\
& \left.+r^{2} d \Omega_{2}^{2}\right]+\left(r^{2}+\frac{z(y)^{2}}{f(r, y)}\right) \frac{d y^{2}}{r^{2}+z(y)^{2}},
\end{aligned}
$$

with

$$
\begin{gathered}
f(r, y)=1-\frac{2 M(r, y)}{\left[r^{2}+z(y)^{2}\right]^{\frac{1}{2}}}, \\
M(r, y)=M_{0}\left\{\frac{\left[r^{2}+z(y)^{2}\right]^{\frac{3}{2}}}{\left(r_{0}^{q}+\left[r^{2}+z(y)^{2}\right]^{\frac{q}{2}}\right)^{\frac{3}{q}}}\right\},
\end{gathered}
$$

and, as we saw, $z(y)=\operatorname{sgn}(y)\left(e^{k|y|}-1\right) / k$. As pointed out by Nakas and Kanti [17], contrary to the Hawking et al. [5] black string, the metric (16) is nonfactorized. And contrary to the geometry studied in Refs. $[17,18]$, there are two horizons here. The mass function (18) is able to provide at most two horizons, an inner $r_{-}$and an outer horizion $r_{+}$, the event horizon properly speaking, since $r_{0}<M_{0}$. Zeros of $g^{r r}\left(r_{ \pm}, y_{0}\right)=f\left(r_{ \pm}, y_{0}\right)=0$ give us the localization of the horizons. As we can see from Fig. 2, both horizons extend to the bulk. This very feature was pointed out by Nakas and Kanti [17,18] and then was argued the "pancake" shape (for a fixed $y=y_{0}$ ) of the event horizon studied by the authors. For a constant mass or $r_{0}=0$, the 


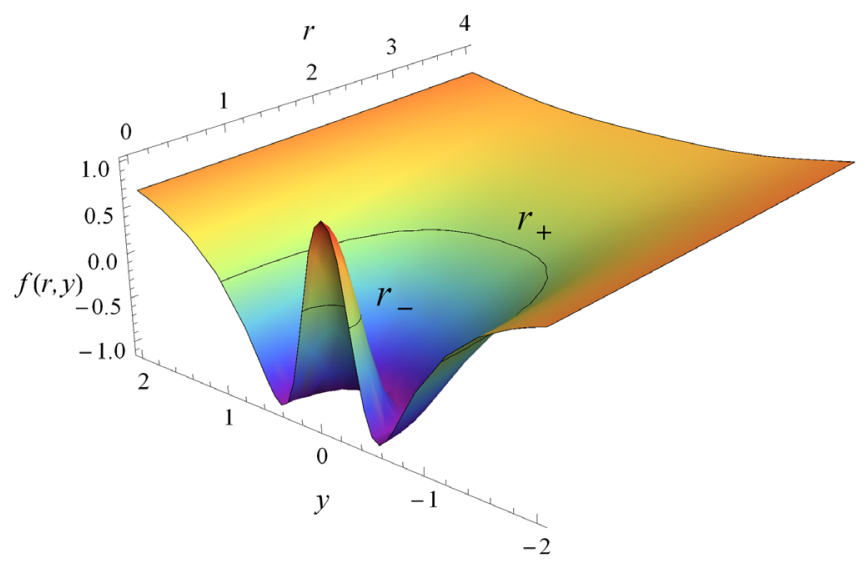

FIG. 2. Zeros of $g^{r r}=f\left(r_{ \pm}, y_{0}\right)=0$ (indicated by the black lines) give us the localization of horizons. The mass function (18) is able to provide two horizons, the inner $r_{-}$and the outer horizon $r_{+}$. As we can see, the horizons get close to the brane (located at $y=0$ ). In this graphic, one uses $k=1, M_{0}=1$, $r_{0}=0.5$ and $q=3$.

"pancake" shape of the horizon (in this case only one) is indicated by $r_{+}^{2}=4 M_{0}^{2}-\left(e^{k\left|y_{0}\right|}-1\right)^{2} / k^{2}$, and $y_{0}$ is simply obtained by making $r_{+}=0$, thus $y_{0}=\ln \left(2 M_{0} k+1\right) / k$ for a constant mass. The value $y_{0}$ means the value of the extra coordinate in which $r_{+}$shrinks exponentially and becomes zero. For our mass function, $y_{0}$ is obtained from $2 k M\left(0, y_{0}\right)-e^{k\left|y_{0}\right|}+1=0$, which give us two values for the edge of the horizons in the extra dimension, namely $y_{0 \pm}$ (related to the outer and inner horizons, respectively).

\section{THE MATTER CONTENT IN THE BULK}

As we can see from the form of the metric element $f(\rho)$ adopted here, the five-dimensional bulk is not an empty or flat spacetime. In order to illustrate this point, following Nakas and Kanti [17] and specifying the bulk energymomentum tensor, one writes the bulk gravitational action as

$$
S_{B}=\int d^{5} x \sqrt{-g}\left(\frac{{ }^{(5)} R}{2 \kappa_{5}^{2}}+\mathcal{L}_{m}^{(B)}\right)
$$

in which $g$ is the metric determinant (from the bulk metric $\left.g_{M N}\right),{ }^{(5)} R$ is the five-dimensional Ricci scalar, $\kappa_{5}^{2}=8 \pi G_{5}$ is defined by the gravitational constant in five dimensions, and $\mathcal{L}_{m}^{(B)}$ is the Lagrangian of the bulk matter content. By means of the variation of (19) with respect to $g_{M N}$, we have the field equations of the bulk, i.e.,

$$
G_{M N}=\kappa_{5}^{2} T_{M N}^{(B)}=-\frac{2 \kappa_{5}^{2}}{\sqrt{-g}} \frac{\delta\left(\mathcal{L}_{m}^{(B)} \sqrt{-g}\right)}{\delta g^{M N}},
$$

where $G_{M N}$ is the five-dimensional Einstein tensor, and $T_{M N}^{(B)}$ is the bulk energy-momentum tensor. From the metric (7), the nonvanishing energy-momentum tensor components in the $(t, \rho, \theta, \phi, \chi)$ coordinates are given by

$$
\begin{aligned}
T^{(B) t}{ }_{t}= & T_{\rho}^{(B) \rho}=\frac{1}{\kappa_{5}^{2}}\left[6 k^{2}+\left(\frac{9 k \cos \chi}{\rho^{2}}-\frac{3}{\rho^{3}}\right) M(\rho)\right. \\
& \left.-\left(\frac{3 k \cos \chi}{\rho}+\frac{3}{\rho^{2}}\right) M^{\prime}(\rho)\right], \\
T_{\theta}^{(B) \theta}= & T^{(B) \phi}{ }_{\phi}=T^{(B) \chi}{ }_{\chi} \\
= & \frac{1}{\kappa_{5}^{2}}\left[6 k^{2}-\left(\frac{6 k^{2} \cos ^{2} \chi}{\rho}-\frac{6 k \cos \chi}{\rho^{2}}\right) M(\rho)\right. \\
& +\left(4 k^{2} \cos ^{2} \chi+\frac{2 k \cos \chi}{\rho}-\frac{2}{\rho^{2}}\right) M^{\prime}(\rho) \\
& \left.-\left(k^{2} \rho \cos ^{2} \chi+2 k \cos \chi+\frac{1}{\rho}\right) M^{\prime \prime}(\rho)\right],
\end{aligned}
$$

where the operator ' means derivative with respect to the function argument. As we can immediately read from Eqs. (21)-(22), the bulk energy-momentum tensor is diagonal in the $(t, \rho, \theta, \phi, \chi)$ coordinates, it can be written as $T^{(B) \mu}{ }_{\nu}=\operatorname{diag}\left(-\rho_{E}, p_{1}, p_{2}, p_{2}, p_{2}\right)$, in which $\rho_{E}$ is the energy density, and $p_{1}$ and $p_{2}$ are pressures. As $p_{1} \neq p_{2}$, thus the bulk spacetime is supported by an anisotropic fluid. Most importantly is the limit of the components of that tensor as $\rho \rightarrow \infty$. That is to say,

$$
\lim _{\rho \rightarrow \infty} \rho_{E}=-\frac{6 k^{2}}{\kappa_{5}^{2}}=\Lambda_{5}
$$

$$
\lim _{\rho \rightarrow \infty} p_{1}=\lim _{\rho \rightarrow \infty} p_{2}=\frac{6 k^{2}}{\kappa_{5}^{2}}=-\Lambda_{5},
$$

in which $\Lambda_{5}$ is the five dimensional cosmological constant. Therefore, the anti-de Sitter feature of the bulk gets evident once again.

In order to see the dependence of (21)-(22) on the extra coordinate, let us write $T^{(B) \mu}{ }_{\nu}$ in the $(t, r, \theta, \phi, y)$ coordinates. Using the transformations (6), we have

$\rho_{E}=-\frac{3 k^{2}}{\kappa_{5}^{2}}\left\{2-(1+\delta)^{-\frac{3+q}{q}} \frac{k M_{0}\left[4(1+\delta)-3 e^{k|y|}\right]}{\left[k^{2} r^{2}+\left(e^{k|y|}-1\right)^{2}\right]^{\frac{3}{2}}}\right\}$,

$$
\begin{aligned}
p_{2}= & \frac{6 k^{2}}{\kappa_{5}^{2}}\left\{1+\frac{k M_{0}\left(e^{k|y|}-1\right)\left(2-e^{k|y|}\right)}{\left[k^{2} r^{2}+\left(e^{k|y|}-1\right)^{2}\right]^{\frac{3}{2}}}\right. \\
& \left.-\frac{k M_{0}\left[\left(4-3 e^{k|y|}-(1+q) \frac{1}{2} e^{2 k|y|}\right) \delta+4 \delta^{2}\right]}{\left[k^{2} r^{2}+\left(e^{k|y|}-1\right)^{2}\right]^{\frac{3}{2}}}\right\},
\end{aligned}
$$

with 


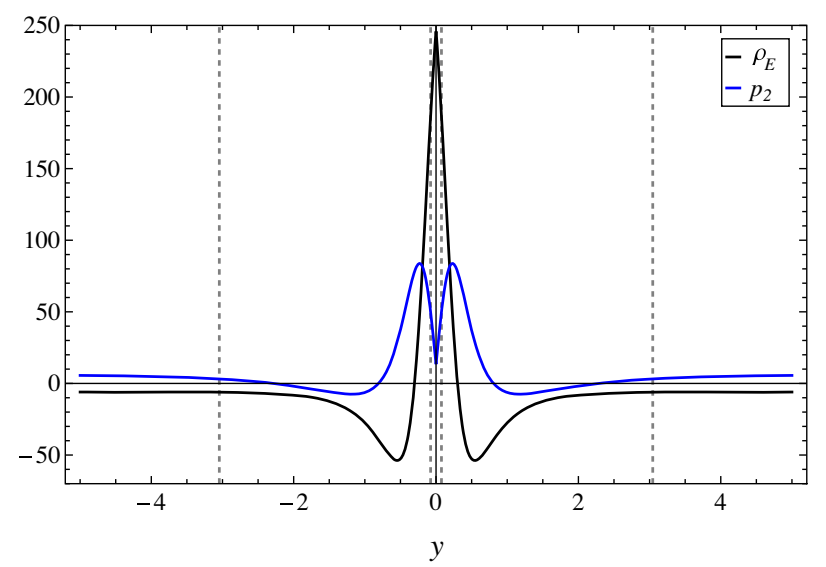

FIG. 3. Components of the bulk energy-momentum tensor and the weak energy violation close to the brane, located at $y=0$. Vertical dashed lines indicate the horizons edges in the bulk (inner $y_{0-}= \pm 0.08$ and outer $y_{0+}= \pm 3.04$ ). In this graphic, one uses $\kappa_{5}=k=1, M_{0}=10, r_{0}=0.1, q=2$ and, most importantly, $r=0.5$.

$$
\delta=\left(\frac{k r_{0}}{\left[k^{2} r^{2}+\left(e^{k|y|}-1\right)^{2}\right]^{\frac{1}{2}}}\right)^{q}
$$

As we can see, for $\delta=0$ or, equivalently, $r_{0}=0$ we have the same results of Ref. [17], and the mass function (18) turns into a constant. In the $(t, r, \theta, \phi, y)$ coordinates, the energy-momentum tensor is not diagonal anymore. Two new off-diagonal components $T_{y}^{(B) r}=e^{2 k|y|} T^{(B) y}$ appear, but both are zero as $y \rightarrow \infty$, as we would expect for an asymptotically anti-de Sitter spacetime. Other components will not be shown here due to the large and cumbersome final forms. The main idea here is just indicate some energy condition violation for the five-dimensional bulk. In particular, as we can see in Fig. 3, the weak energy condition is violated close to the brane, i.e., $\rho_{E}<0$ and $\rho_{E}+p_{2}<0$. Moreover, Fig. 4 shows that for $r=0$ and $y=0$, $p_{2} / \rho_{E}=-1$, which is the equation of state of a de Sitter spacetime. As we will see, this indicates that a de Sitter core is inside the event horizon and it is entirely on the brane, not in the bulk. For a five-dimensional de Sitter space one has $g_{t t}=-\left(1-r^{2} / \alpha^{2}\right)$, with $\alpha$ constant, and from Eq. (16), for small values of the radial coordinate $r$, we have $g_{t t} \simeq-\left(1-\beta r^{2}\right)$, with $\beta$ constant, only for $y=0$, that is, outside the extra dimension.

\section{THE BRANE PERSPECTIVE}

\section{A. Gravitational field equations on the brane}

With $y=z=0$ in the metric (16), we have a fourdimensional RBH. But would it be a RBH in a brane context? For sure in that context the gravitational field equations are not the Einsteinian equations. In the fourdimensional world, the brane, the field equations are very

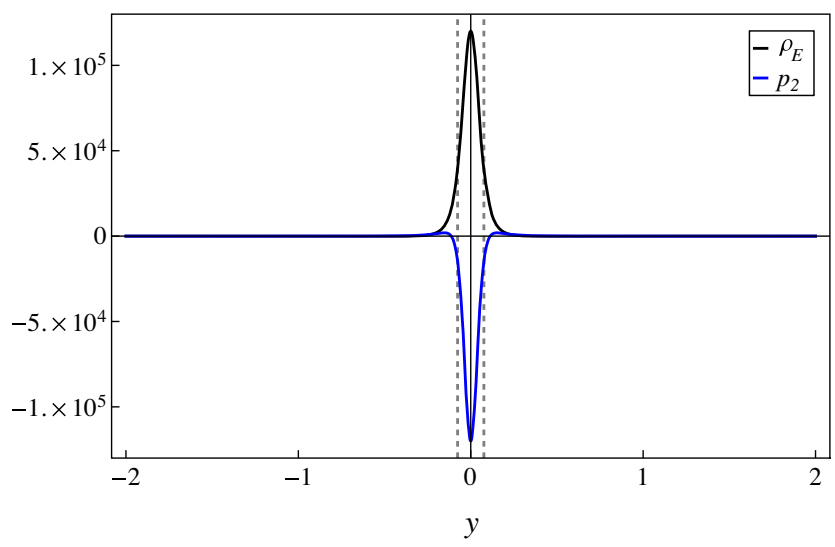

FIG. 4. Components of the bulk energy-momentum tensor for $r=0$ on the brane. The vertical dashed lines indicate the inner horizon limit in the bulk $\left(y_{0-}= \pm 0.08\right)$. In this graphic, one uses $\kappa_{5}=k=1, M_{0}=10, r_{0}=0.1$ and $q=2$. For $y=0$, we see a de Sitter equation of state $\left(p_{2} / \rho_{E}=-1\right)$.

different ones. They were deduced from the Gauss and Codacci equations, according to the seminal work of Shiromizu, Maeda, and Sasaki [19]. I adopt here a scenario like that one. In such a scenario, the four-dimensional brane $\left(\Sigma, h_{M N}\right)$ is embedded into a five-dimensional bulk $\left(\mathcal{M}, g_{M N}\right)$, and a normal and unit vector to the brane is written as $n^{M}=h^{M}{ }_{y}$. Therefore, the induced metric on the brane reads $h_{M N}=g_{M N}-n_{M} n_{N}$.

Following Nakas and Kanti $[17,18]$, one defines the total energy-momentum tensor as

$$
T_{M N}=T_{M N}^{(B)}+h^{\mu}{ }_{M} h^{\nu}{ }_{N} T_{\mu \nu}^{(b r)} \delta(y),
$$

with the brane energy-momentum tensor, like many brane world scenarios, given by

$$
T^{(b r)}=-\lambda h_{\mu \nu}+\tau_{\mu \nu} .
$$

The constant $\lambda$ is the brane tension, some sort of vacuum energy on the brane, and $\tau_{\mu \nu}$ regards all matter fields on the four-dimensional spacetime. As we will see, the tensor $\tau_{\mu \nu}$ vanishes, and the RBH on the brane will be supported by the bulk geometrical influence on the brane. Another important point here is that the total energy-momentum tensor (28) is different from that one chosen in Ref. [19]. The bulk energy-momentum tensor is not described by a cosmological constant term. As we saw in the previous section, an exotic field ensures the asymptotic anti-de Sitter behavior for the bulk spacetime instead of a five dimensional cosmological constant.

In order to show that $\tau_{\mu \nu}=0$ in the brane context studied here, one uses Israel's junction condition [43] at $y=0$ and a very important result for the extrinsic curvature $K_{\mu \nu}$, namely 


$$
\left[K_{\mu \nu}\right]=-\kappa_{5}^{2}\left(T_{\mu \nu}^{(b r)}-\frac{1}{3} h_{\mu \nu} T^{(b r)}\right)
$$

where $T^{(b r)}$ is the trace of the energy-momentum tensor on the brane. The extrinsic curvature is defined as

$$
K_{\mu \nu}=h^{A}{ }_{\mu} h^{B}{ }_{\nu} \nabla_{A} n_{B},
$$

with $n^{A}=\left(0,0,0,0,1 / \sqrt{g_{y y}(y=0)}\right)$, and the bracket notation means

$$
[X]=\lim _{y \rightarrow 0^{+}} X-\lim _{y \rightarrow 0^{-}} X=X^{+}-X^{-}
$$

The induced metric $h_{\mu \nu}$ on the brane in the $(t, r, \theta, \phi)$ coordinates is written as

$d s^{2}=-\left(1-\frac{2 m(r)}{r}\right) d t^{2}+\left(1-\frac{2 m(r)}{r}\right)^{-1} d r^{2}+r^{2} d \Omega_{2}^{2}$

with

$$
m(r)=M(r, 0)
$$

and it clearly satisfies $\left[h_{\mu \nu}\right]=0$. With the aid of (16), then the extrinsic curvature reads simply

$$
K_{\mu \nu}=-k \frac{d|y|}{d y} h_{\mu \nu}
$$

and follows that $K=-4 k \frac{d|y|}{d y}$. With the extrinsic curvature calculated, the definition (30) (contracted with $h^{\mu \nu}$ ) leads to

$$
T^{(b r)}=\frac{3}{\kappa_{5}^{2}}[K] .
$$

Therefore, Eq. (30) can be rewritten as

$$
T_{\mu \nu}^{(b r)}=-\frac{1}{\kappa_{5}^{2}}\left(\left[K_{\mu \nu}\right]-[K] h_{\mu \nu}\right)=-\frac{6 k}{\kappa_{5}^{2}} h_{\mu \nu},
$$

in which the $\mathbb{Z}_{2}$ symmetry was used. From the above result and Eq. (29), one concludes that $\tau_{\mu \nu}=0$, that is to say, there are no sources or matter fields on the brane. Besides, the brane tension is related to the anti-de Sitter curvature by means of $\lambda=6 k / \kappa_{5}^{2}>0$. Then the RBH geometry should be produced by the bulk influence on the brane (this point will be emphasized again below).

In order to describe the bulk influence on the brane, the field equations induced on the brane are necessary, equations with components that deforms the brane generating then a black hole. As I said, the induced field equations were deduced by Shiromizu, Maeda, and Sasaki [19]. Such field equations are given by

$$
\begin{aligned}
G_{\mu \nu}= & \frac{2 \kappa_{5}^{2}}{3}\left(h^{M}{ }_{\mu} h_{\nu}^{N} T_{M N}^{(B)}+\left[T_{M N}^{(B)} n^{M} n^{N}-\frac{T^{(B)}}{4}\right] h_{\mu \nu}\right) \\
& +K K_{\mu \nu}-K_{\mu}{ }^{\alpha} K_{\nu \alpha}-\frac{1}{2} h_{\mu \nu}\left(K^{2}-K^{\alpha \beta} K_{\alpha \beta}\right)-E_{\mu \nu},
\end{aligned}
$$

where $G_{\mu \nu}$ is the four-dimensional Einstein tensor, $T^{(B)}$ is the trace of the bulk energy-momentum tensor, and $E_{\mu \nu}$ is the so-called "electric" part of the five-dimensional Weyl tensor $\left(C^{A}{ }_{B C D}\right)$ projected onto the brane. Its form is

$$
E_{\mu \nu}=C_{B C D}^{A} n_{A} n^{C} h_{\mu}^{B} h_{\nu}^{D} .
$$

It is worth pointing out that $E^{\mu}{ }_{\mu}=0$, i.e., it is a traceless tensor. Therefore, from the metric (16), with the mass function (18), $E_{\mu \nu}$ reads

$$
\left.E_{\mu \nu}\right|_{y \rightarrow 0}=\frac{M_{0} \mathcal{E}}{r^{3}}\left(\begin{array}{cccc}
-h_{t t} & & & \\
& -h_{r r} & & \\
& & h_{\theta \theta} & \\
& & & h_{\phi \phi}
\end{array}\right) \text {, }
$$

with

$$
\mathcal{E}=\frac{\left[2-(1+q)\left(\frac{r_{0}}{r}\right)^{q}\right]}{2\left[1+\left(\frac{r_{0}}{r}\right)^{q}\right]^{\frac{2 q+3}{q}}} .
$$

For $r_{0}=0, M(r, 0)=m(r)=M_{0}$ and $\mathcal{E}=1$, then we recover the results of Ref. [17]. Moreover, $E_{\mu \nu}$ is also regular due to the mass function, that is to say, $\lim _{r \rightarrow 0} E_{\mu \nu}=0$.

The terms of the gravitational field equations (38) that contain the extrinsic curvature, with the aid of Eq. (35), result in

$$
\begin{gathered}
K K_{\mu \nu}-K_{\mu}^{\alpha} K_{\nu \alpha}-\frac{1}{2} h_{\mu \nu}\left(K^{2}-K^{\alpha \beta} K_{\alpha \beta}\right) \\
=8 \pi G \tau_{\mu \nu}+\kappa_{5}^{4}\left(\pi_{\mu \nu}-\frac{\lambda^{2}}{12} h_{\mu \nu}\right),
\end{gathered}
$$

with $G=\kappa_{5}^{4} \lambda / 48 \pi=1$ playing the role of the effective gravitational constant on the brane, and

$\pi_{\mu \nu}=-\frac{1}{4} \tau_{\mu \alpha} \tau_{\nu}{ }^{\alpha}+\frac{1}{12} \tau \tau_{\mu \nu}+\frac{1}{8} \tau_{\alpha \beta} \tau^{\alpha \beta} h_{\mu \nu}-\frac{\tau^{2}}{24} h_{\mu \nu}$.

By using the above results, namely Eqs. (42) and (43), we are able to rewrite the field equations in terms of an effective energy-momentum tensor, i.e., 


$$
G_{\mu \nu}=8 \pi G\left(T_{\mu \nu}^{(\mathrm{eff})}+\tau_{\mu \nu}\right)+\kappa_{5}^{4}\left(\pi_{\mu \nu}-\frac{\lambda^{2}}{12} h_{\mu \nu}\right)-E_{\mu \nu} .
$$

It is worth emphasizing that $\pi_{\mu \nu}=0$ because, as we saw, $\tau_{\mu \nu}=0$. The above field equations then present an effective energy-momentum tensor (diagonal tensor) given by

$$
T_{\mu \nu}^{(\mathrm{eff})}=\left.\frac{2}{3 k}\left(T_{\mu \nu}^{(B)}+\left[T_{y y}^{(B)}-\frac{T^{(B)}}{4}\right] h_{\mu \nu}\right)\right|_{y=0},
$$

calculated at $y=0$. The explicit form of (45) is then

$$
\begin{aligned}
& T_{\mu \nu}^{(\mathrm{eff})}=\frac{1}{\kappa_{5}^{2} k}\left[\frac{M_{0}}{r^{3}}\left[\begin{array}{lll}
-\mathcal{T}_{1} h_{t t} & & \\
& -\mathcal{T}_{1} h_{r r} & \\
& \mathcal{T}_{2} h_{\theta \theta} & \\
& & \mathcal{T}_{2} h_{\phi \phi}
\end{array}\right)\right. \\
&\left.+3 k^{2} h_{\mu \nu}\right],
\end{aligned}
$$

with

$$
\begin{gathered}
\mathcal{T}_{1}=\frac{\left[12\left(\frac{r_{0}}{r}\right)^{2 q}+(11-q)\left(\frac{r_{0}}{r}\right)^{q}+2\right]}{2\left[1+\left(\frac{r_{0}}{r}\right)^{q}\right]^{\frac{2 q+3}{q}}}, \\
\mathcal{T}_{2}=-\frac{\left[12\left(\frac{r_{0}}{r}\right)^{2 q}-5(1+q)\left(\frac{r_{0}}{r}\right)^{q}-2\right]}{2\left[1+\left(\frac{r_{0}}{r}\right)^{q}\right]^{2 q+3}} .
\end{gathered}
$$

Once again, for $r_{0}=0, \mathcal{T}_{1}=\mathcal{T}_{2}=1$, and we recover the Nakas and Kanti results [17] when the mass parameter is a positive constant.

\section{B. Four-dimensional regular black hole}

The effective energy-momentum tensor (46) is also regular on the brane. Calculating it, one has

$$
\lim _{r \rightarrow 0} T_{\nu}^{(\mathrm{eff}) \mu}=\frac{3}{\kappa_{5}^{2}}\left(k-\frac{2 M_{0}}{k r_{0}^{3}}\right)
$$

for all nonzero components. Assuming the field equations (44) for $r \rightarrow 0$, we have $G^{\mu}{ }_{\nu}=-\frac{6 M_{0}}{r_{0}^{3}} h^{\mu}{ }_{\nu}$, suggesting then a de Sitter core, that which prevents the singularity issue, inside the hole. Thus

$$
\ell_{d S}=\left(\frac{r_{0}^{3}}{2 M_{0}}\right)^{\frac{1}{2}}
$$

with $\ell_{d s}$ playing the role of the de Sitter curvature radius. In this case, we have a de Sitter core inside the event horizon like well-known RBHs (as pointed out in Ref. [24]).

As we know, the gravitational field equations can provide the four-dimensional Einstein tensor. Calculating the right side of (44), one has

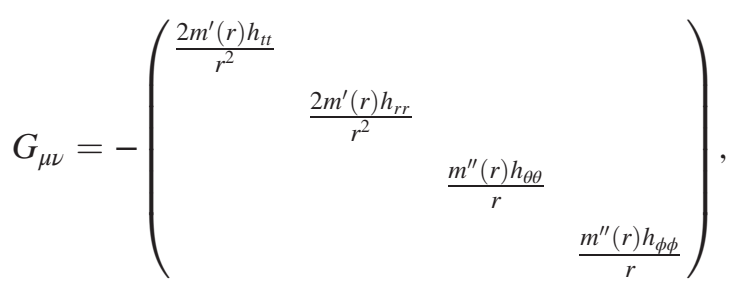

which is the same Einstein tensor for a $\mathrm{RBH}$ in the general relativity context with $m(r)$ given by Eq. (34). But as our brane is a vacuum spacetime, the origin or source for that RBHs comes from the five-dimensional bulk and its geometrical influence on the brane. With the Einstein tensor indicated above, we have then well-known RBHs on the brane, whether the Bardeen RBH (for $q=2$ ) or the Hayward RBH (for $q=3$ ).

\section{FINAL REMARKS}

When applied to the gravitational phenomenon, braneworld models are richer than the Einsteinian context. Like in the general relativity context, brane RBHs are even possible. Here a recent approach, due to Nakas and Kanti [17], was adopted. From the bulk spacetime to the brane spacetime (calculating the induced gravitational field equations), the geometries presented here are regular both in the five-dimensional and on the four-dimensional world, contrary to the so-called black string or even the Nakas and Kanti geometries, which are singular on the brane. Above all, the geometries obtained in this article are fivedimensional RBHs because their horizons extend to the extra dimension, and, interestingly, the de Sitter core - that which precludes the central singularity-is entirely on the four-dimensional brane.

From the induced field equations on the brane, we saw that the four-dimensional brane is a vacuum spacetime. Thus, the source of the RBH on the brane is in the fivedimensional bulk. Then the bulk influence on the brane is geometrical one. An important point in this article regards well-known RBHs in the general relativity context, like the Bardeen and Hayward RBH, which are found in the brane context adopted here. 
[1] L. Randall and R. Sundrum, A Large Mass Hierarchy from A Small Extra Dimension, Phys. Rev. Lett. 83, 3370 (1999).

[2] L. Randall and R. Sundrum, An Alternative to Compactification, Phys. Rev. Lett. 83, 4690 (1999).

[3] R. Maartens and K. Koyama, Brane-world gravity, Living Rev. Relativity 13, 5 (2010).

[4] T. Clifton, P. G. Ferreira, A. Padilla, and C. Skordis, Modified gravity and cosmology, Phys. Rep. 513, 1 (2012).

[5] A. Chamblin, S. W. Hawking, and H. S. Reall, Brane world black holes, Phys. Rev. D 61, 065007 (2000).

[6] R. Casadio, A. Fabbri, and L. Mazzacurati, New black holes in the brane world?, Phys. Rev. D 65, 084040 (2002).

[7] K. A. Bronnikov, V. N. Melnikov, and H. Dehnen, On a general class of brane world black holes, Phys. Rev. D 68, 024025 (2003).

[8] C. Molina and J. C. S. Neves, Black holes and wormholes in AdS branes, Phys. Rev. D 82, 044029 (2010).

[9] C. Molina and J. C. S. Neves, Wormholes in de Sitter branes, Phys. Rev. D 86, 024015 (2012).

[10] J. C. S. Neves and C. Molina, Rotating black holes in a Randall-Sundrum brane with a cosmological constant, Phys. Rev. D 86, 124047 (2012).

[11] F. S. N. Lobo, A general class of braneworld wormholes, Phys. Rev. D 75, 064027 (2007).

[12] C. Barcelo and M. Visser, Brane surgery: Energy conditions, traversable wormholes, and voids, Nucl. Phys. B584, 415 (2000).

[13] N. Dadhich, R. Maartens, P. Papadopoulos, and V. Rezania, Black holes on the brane, Phys. Lett. B 487, 1 (2000).

[14] A. N. Aliev and A. E. Gumrukcuoglu, Charged rotating black holes on a 3-brane, Phys. Rev. D 71, 104027 (2005).

[15] J. C. S. Neves, Note on regular black holes in a brane world, Phys. Rev. D 92, 084015 (2015).

[16] S. Creek, R. Gregory, P. Kanti, and B. Mistry, Braneworld stars and black holes, Classical Quant. Grav. 23, 6633 (2006).

[17] T. Nakas and P. Kanti, Localized brane-world black hole analytically connected to an $\mathrm{AdS}_{5}$ boundary, Phys. Lett. B 816, 136278 (2021).

[18] T. Nakas and P. Kanti, Analytic and exponentially localized brane-world Reissner-Nordström-AdS solution: A topdown approach, arXiv:2105.06915 [Phys. Rev. D (to be published)].

[19] T. Shiromizu, K. i. Maeda, and M. Sasaki, The Einstein equation on the 3-brane world, Phys. Rev. D 62, 024012 (2000).

[20] J. M. Bardeen, in Proc. Int. Conf. GR5, Tbilisi, Vol. 174 (Tbilisi University Press, Tbilisi, U.S.S.R., 1968).

[21] S. Ansoldi, Spherical black holes with regular center: A Review of existing models including a recent realization with Gaussian sources, arXiv:0802.0330.

[22] J. P. S. Lemos and V. T. Zanchin, Regular black holes: Electrically charged solutions, Reissner-Nordström outside a de Sitter core, Phys. Rev. D 83, 124005 (2011).
[23] I. Dymnikova, Spherically symmetric space-time with the regular de Sitter center, Int. J. Mod. Phys. D 12, 1015 (2003).

[24] J. C. S. Neves and A. Saa, Regular rotating black holes and the weak energy condition, Phys. Lett. B 734, 44 (2014).

[25] A. D. D. Masa, E. S. de Oliveira, and V. T. Zanchin, Stability of regular black holes and other compact objects with a charged de Sitter core and a surface matter layer, Phys. Rev. D 103, 104051 (2021).

[26] R. Wald, General Relativity (University of Chicago, Chicago, 1984).

[27] I. Dymnikova, Vacuum nonsingular black hole, Gen. Relativ. Gravit. 24, 235 (1992).

[28] I. Dymnikova, De Sitter-Schwarzschild black hole: Its particle-like core and thermodynamical properties, Int. J. Mod. Phys. D 05, 529 (1996).

[29] S. A. Hayward, Formation and Evaporation of Regular Black Holes, Phys. Rev. Lett. 96, 031103 (2006).

[30] J. C. S. Neves, Deforming regular black holes, Int. J. Mod. Phys. A 32, 1750112 (2017).

[31] A. Smailagic and E. Spallucci, Kerrr black hole: The lord of the string, Phys. Lett. B 688, 82 (2010).

[32] C. Bambi and L. Modesto, Rotating regular black holes, Phys. Lett. B 721, 329 (2013).

[33] B. Toshmatov, B. Ahmedov, A. Abdujabbarov, and Z. Stuchlik, Rotating regular black hole solution, Phys. Rev. D 89, 104017 (2014).

[34] M. Azreg-Aïnou, Generating rotating regular black hole solutions without complexification, Phys. Rev. D 90, 064041 (2014).

[35] R. V. Maluf and J. C. S. Neves, Thermodynamics of a class of regular black holes with a generalized uncertainty principle, Phys. Rev. D 97, 104015 (2018).

[36] J. C. S. Neves and A. Saa, Accretion of perfect fluids onto a class of regular black holes, Ann. Phys. (Amsterdam) 420, 168269 (2020).

[37] K. A. Bronnikov, Regular magnetic black holes and monopoles from nonlinear electrodynamics, Phys. Rev. D 63, 044005 (2001).

[38] L. Modesto and P. Nicolini, Charged rotating noncommutative black holes, Phys. Rev. D 82, 104035 (2010).

[39] S. G. Ghosh, D. V. Singh, and S. D. Maharaj, Regular black holes in Einstein-Gauss-Bonnet gravity, Phys. Rev. D 97, 104050 (2018).

[40] W. C. Hernandez, Material sources for the kerr metric, Phys. Rev. 159, 1070 (1967).

[41] E. Ayon-Beato and A. Garcia, The Bardeen model as a nonlinear magnetic monopole, Phys. Lett. B 493, 149 (2000).

[42] R. V. Maluf and J. C. S. Neves, Bardeen regular black hole as a quantum-corrected Schwarzschild black hole, Int. J. Mod. Phys. D 28, 1950048 (2019).

[43] W. Israel, Singular hypersurfaces and thin shells in general relativity, Nuovo Cimento B 44, 1 (1966); Erratum, Nuovo Cimento B 48, 463 (1967). 